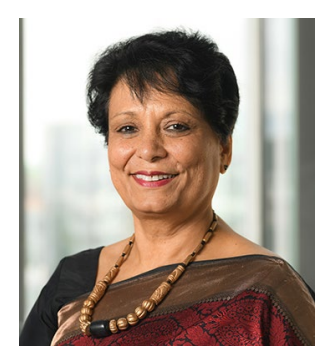

\title{
COVAX can still end COVID-19 vaccine apartheid
}

\author{
COVAX emerged as a key mechanism to advance COVID-19 vaccine equity. To fully succeed, it \\ needs support that extends beyond vaccine donations, argues Anuradha Gupta.
}

Credit: Tony Noël

$\mathrm{S}$ ince the COVID-19 pandemic began, the pressure on government leaders at all levels has been extraordinary. To protect lives and livelihoods during an unprecedented global crisis, elected officials and other public servants in areas as diverse as commerce, education and transportation have been thrust into the public health sphere. Their constitutional duty to protect their citizens enabled a swift roll-out of COVID-19 vaccines to contain serious illness, hospitalization and deaths. But also it gave rise to a phenomenon that we know now to be counterproductive: vaccine hoarding. The best way to break COVID-19 is to balance national interests and global need, and to ensure that at least high-risk and vulnerable populations in every part of the world are equitably regarded. Vaccine 'apartheid', which puts a large number of countries at the back of the queue, means the pandemic might drag on longer than necessary.

For some government leaders, pursuing a strategy of vaccine equity abroad to lower COVID-19 infection rates at home may sound counterintuitive. How can governments protect their own citizens and keep society running if they are giving away vaccines to people on the other side of the globe? But this is exactly what the latest research indicates: to end the pandemic, priority populations in lower-income countries must get their first dose of a COVID-19 vaccine before general populations in higher-income countries get their third or fourth. To stop new cases and new variants such as Omicron will require breadth in vaccination coverage, not depth; reaching more people, especially those at higher risk, with at least two doses.

Is vaccine equity a utopian vision? Is it realistic to expect that scarce vaccines would be willingly shared by countries that control their development and production? As deputy CEO of Gavi, the Vaccine Alliance, I have seen important lessons emerge from the work of COVAX - a multilateral solution built specifically to advance equitable access to COVID-19 vaccines. A collaboration between Gavi, the United Nations Children's
Fund (UNICEF), the World Health Organization (WHO) and the Coalition for Epidemic Preparedness Innovations (CEPI), COVAX has now shipped more than one billion doses of COVID-19 vaccines to 144 economies around the world - the overwhelming majority to lower-income countries. But many countries had to wait far too long for quantities of vaccine that were insufficient to achieve the equitable coverage necessary to end this pandemic. Meanwhile, the emergence of highly transmissible Omicron might reverse recent progress in bridging the vaccine chasm.

Predictably, the Omicron storm has seen domestic pressures rise once again, which could lead to a dwindling of vaccine donations to lower-income countries. But with vaccine manufacturing capacity at an all-time high and global supply constraints easing, the world needs to do better at making sure vaccines are no longer hoarded - and are allowed to reach all countries, and priority populations within countries, in a timely and efficient manner. Failure to do so would accentuate a sense of betrayal on the part of communities, further heighten mistrust of governments, and fuel misperceptions and rumours. After all, the equitable distribution of effective vaccines is only the first step: communities must come forward to receive those vaccines. Building community demand on the basis of trust and transparency is crucial to vaccine equity.

The question remains of whether COVAX could have delivered more. The answer is an unequivocal 'yes': as a mechanism, it is capable of delivering at scale, but it needs timely financing for an agile response in a fast-changing environment. COVAX needs to rapidly provide countries the best vaccine products and sufficient quantities, as well as nimble support to convert vaccines into vaccinations as quickly as possible. Lower-income economies need transparency on vaccine-product choices and supply schedules; they need vaccines with long shelf lives; and they need advance information for long-term planning, to safely and effectively protect the maximum number of people, beginning with high-risk groups.

Further, the majority of donations of doses of COVID-19 vaccines to lower-income countries have not included syringes, diluent or freight costs. As highlighted in a joint statement in November 2021 by the African Vaccine Acquisition Trust (AVAT), the Africa Centres for Disease Control and Prevention (Africa CDC) and COVAX, donated doses must be accompanied with all essential ancillaries if they are to fulfil their life-saving potential. Otherwise, we are creating a risk of redeployment of ancillaries meant for routine immunization. It would be catastrophic if childhood vaccination drops, as individuals who are the most marginalized would lose access to life-saving vaccines, exacerbating inequity and increasing child deaths among this group. It is unfortunate that from 2019 to 2020 , the number of 'zero-dose' children, who have not received even a single vaccine shot, rose by $30 \%$ to nearly 14 million in lower-income countries supported by Gavi.

The World Bank's latest Global Economic Prospects report, released on 11 January 2022, is a brutal reminder of our shared economic challenges, as we face growing income inequality not only across but also within countries. The devastating socioeconomic and health effects of vaccine apartheid have repercussions far into the future, especially for women and children. Vaccine equity cannot be postponed any longer. Otherwise, at the beginning of 2023, we might find ourselves in the same position - but confronting a wave of Omega rather than Omicron. Vaccine equity is not just a lofty ideal; it is our best hope for ending the pandemic and reclaiming public health gains.

\section{Anuradha Gupta ${ }^{凶}$ \\ Gavi, the Vaccine Alliance, Geneva, Switzerland. \\ $凶_{e-m a i l: m e d i a @ g a v i . o r g}$}

Published online: 31 January 2022 https://doi.org/10.1038/s41562-022-01308-8

Competing interests

The author declares no competing interests. 